\title{
Where Your Data Is Going and Where It's Been
}

Jane M. Quigley, RN, Plymouth Meeting, Pennsylvania

\section{Key Words}

Data collection, health care, oncology, REMS

\begin{abstract}
Collecting data is time-consuming and expensive. Electronic transactions, including electronic medical records and the unification of many billing procedures, have transformed the rate at which data are able to be extracted. Although obstacles remain, the rate at which data are de-identified, collected, and aggregated will help improve safety and standards of care for oncology patients. Everyone participating in the care of oncology patients must understand how important data are as the quality initiatives and performance metrics are numerous and growing; these initiatives cannot be successful without timely, accurate, and quantifiable data that address the continuum of oncology care. (JNCCN 2011;9[Suppl 3]:S10-S12)
\end{abstract}

“... time is lost, confusion results, and money is spent ...”

This quote, expressed in 1917 by a Connecticut manufacturing manager, referred to the telephone. Imagine his thoughts now, when a company such as Walmart alone handles more than 1 million customer transactions electronically every hour. ${ }^{1}$

Today, the amount of data flowing through every technical medium is enormous. Health care information alone

From SDI, Plymouth Meeting, Pennsylvania.

Submitted December 9, 2010; accepted for publication January 10, 2011.

The author has disclosed that she has no financial interests, arrangements, or affiliations with the manufacturers of any products discussed in this article or their competitors.

The ideas and viewpoints expressed in this article are those of the author and do not necessarily represent any policy, position, or program of NCCN.

Correspondence: Jane M. Quigley, RN, SDI, 1 SDI Drive, Plymouth Meeting, PA 19462. E-mail: jquigley@sdihealth.com is voluminous, and numerous providers, federal and state governments, cooperative groups, physicians, laboratories, hospitals, data companies, and pharmacies see a lot of it.

No one is invisible when it comes to health care data; patients, physicians, hospitals, and pharmacies all generate a trail of data footprints that are gathered and analyzed by someone every day. This volume of data is expected to grow exponentially in the coming years. The streamlining and unification of billing forms, e-prescribing, electronic medical records, and practice management systems used for scheduling and billing will all amplify this growth.

Health care technology has the goal of improving safety through better diagnostic, treatment, and management techniques for all participants in the health care continuum. To meet this goal, rules governing privacy had to be amended to secure personal information. The federal government responded in 1996 with the Health Insurance Portability and Accountability Act (HIPAA). HIPAA was created to protect patients, providers, and clearinghouses that process medical data and integrate billing standards. HIPAA provides oversight of all personal health information. The federal government furthered that initiative with the Health Information Technology for Economic and Clinical Health (HITECH) Act of 2009.

HIPAA also required that all medical insurance forms be universal. The HCFA 1500 form is used by all insurance companies across the United States to process medical insurance claims. Contained within the HCFA 1500 form are details regarding the physician, International classification of diseases-ninth revision (ICD-9) diagnosis codes, current procedural terminology (CPT) codes, and Healthcare Common Procedure Coding System (HCPCS) codes. These forms also contain National Provider Identifier (NPI) information, which allows all of this information to be tied back to the rendering 


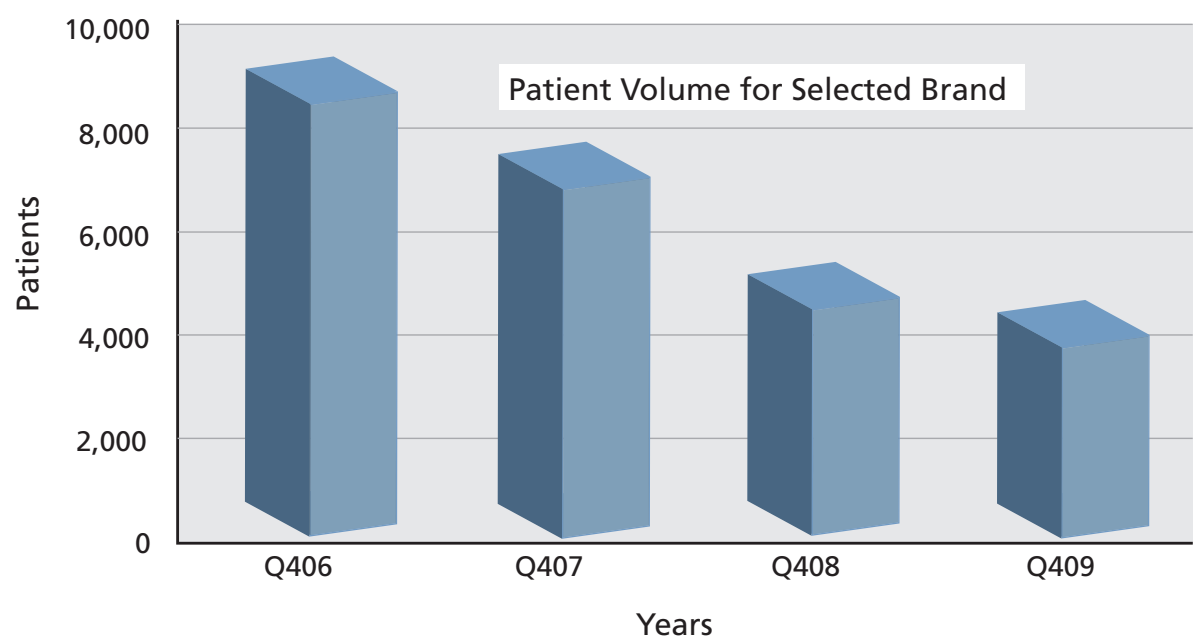

Figure 1 Blinded use of a product with a REMS program.

physician, hospital, and pharmacy. The amount of health care data has grown exponentially since the enactment of HIPAA.

As technology improves, so does the need for protecting health information. Some providers, physicians, patients, and pharmacies may be tacitly aware that their data are "out there," but few recognize how often the data are analyzed and for what purposes. Safety, clinical trial development, and costs are often the main objectives of data analysis. These data also have allowed physicians to be located who are associated with a large volume of certain patient types, and thereby to be identified as "key opinion leaders." These thought leaders often influence how standards of care are established and change over time with the dissemination and facilitation of new and emerging information.

Additionally, the FDA Amendments Act of 2007 (FDAAA) gave the FDA authority to require a Risk Evaluation and Mitigation Strategy (REMS) for certain drugs with a high risk potential. As part of these new provisions, ongoing postmarketing safety data for numerous products with a REMS program must be collected. Currently, more than 150 medications are associated with a REMS program, including everything from asthma therapies to narcotics. Executing a REMS program requires a lot of data on both patients and providers. However, most companies with a drug requiring a REMS program are not able to "see" their data because the data are proprietary to the FDA.

REMS programs serve as a registry of patients, physicians, hospitals, and pharmacies dispensing a REMSrequired product. Figure 1 lists a blinded example of a product currently requiring a REMS program. As depicted, patient numbers have been decreasing over time because of newer restrictions on its therapeutic use.

\section{Ask the Data the Right Questions}

With so much quantitative and qualitative data available, it is more important than ever to know how to "ask the data the right questions" to obtain relevant and valid results.

Physician-level data are ubiquitous. Oncology patients, providers, clinical research organizations, the federal government, and pharmaceutical companies have unprecedented access to this information. They can see how many abstracts a physician coauthored through ASCO, whether a doctor is part of an academic or community setting, or if a physician is up-to-date on subspecialty certification through the American Board of Internal Medicine. Data are now structured in formats that are easily queried and provide unparalleled insights into patterns of care, costs associated with care, and health outcomes. Understanding which of these many data elements are most relevant and understanding the underlying disease state are becoming more important than ever given the interplay among physicians, pharmacies, and hospitals.

\begin{tabular}{|llll|}
\hline Table 1 & $\begin{array}{l}\text { Data on Drug Use for Patients With } \\
\text { Non-Small Cell Lung Cancer }\end{array}$ \\
$\begin{array}{llll}\text { Total } \\
\text { Patients }\end{array}$ & $\begin{array}{l}\text { No. on } \\
\text { Pemetrexed }\end{array}$ & $\begin{array}{l}\text { No. on } \\
\text { Docetaxel }\end{array}$ & $\begin{array}{l}\text { No. on } \\
\text { Tarceva }\end{array}$ \\
\hline 3666 & 2548 & 3336 & 2218 \\
\hline
\end{tabular}


Quigley

Table 2 Insurance Denial Data for Albumin-Bound Paclitaxel

\begin{tabular}{|llll|}
\hline Adjustment Category & Adjustment Reason & Claims & Physicians \\
\hline Contractual obligations & Charges exceed the contracted/legislated fee arrangement & 8752 & 604 \\
& $\begin{array}{l}\text { Payment adjusted because of the impact of prior payors } \\
\text { adjudication, including payments and/or adjustments }\end{array}$ & 785 & 5161 \\
Patient responsibility & Coinsurance amount & 392 & 404 \\
& Payment based on authorized amount & 1339 & 301 \\
Other adjustments & Payment adjusted because of the impact of prior payors & 356 \\
& adjudication, including payments and/or adjustments & 122 \\
& The number of days or units of service our acceptable maximum & 356 \\
\hline
\end{tabular}

Assessing hospitals, hospital-associated practices, and large multi-specialty physician practices is critical in understanding oncology drug use and costs of therapy. Many large academic medical centers can employ more than 1000 oncology specialists. Understanding how these specialists practice is necessary to analyze how standards of care are evolving.

Observing how a large community-based oncology practice treats their patients, and evaluating practice patterns in these clinics and in academic medical centers allows for a complete understanding of practice patterns at a State and National level. Understanding these treatment patterns allows for more streamlined and expedient deployment of health care services and enables assessment of appropriate populations for inclusion in clinical trials. Care of cancer patients is changing. Understanding and incorporating hospital-based treatments, radiation therapy, and oral and intravenous oncologic use, including specialty pharmacy distribution, is a challenge. Proper integration of this information to accurately measure patient incidence, prevalence, and treatment patterns is more important than ever given the growing costs of health care.

Table 1 shows how integrating physician, ICD-9 code, and specialty pharmacy data is critical in understanding patient care and drug use for lung cancer. To accurately understand treatment paradigms within non-small cell lung cancer, it is critical to integrate and understand these disparate data sources. Without addressing all facets within the health care system, misinformation and additional costs are likely.

Physicians, nurses, and pharmacists must be aware of the burgeoning layers associated with treating oncology patients. Understanding which therapies are used for which tumor types, and which are used as initial therapy or for treatment-refractory patients is a complex process. Incorporating data on which of these therapies is coming from a specialty pharmacy or requires a preauthorization from the insurance company is a formidable additional task. When the appropriate steps are not taken to address the special circumstances surrounding the use of certain oncology therapeutics, rebilling and additional work are required. Table 2 lists an example of how remittance data can show providers and physicians which drugs are being denied by the insurance company and for what reasons. Addressing these reimbursement issues is paramount for providers and payors.

\section{Where Will Your Data Go Next?}

Collecting data is time-consuming and expensive. Electronic transactions, including electronic medical records and the unification of many billing procedures, have transformed the rate at which data are able to be extracted. Although obstacles remain, the rate at which data are de-identified, collected, and aggregated will help improve safety and standards of care for oncology patients.

Everyone participating in the care of oncology patients must understand how important data are. Quality initiatives and performance metrics are numerous and growing, and cannot be successful without timely, accurate, and quantifiable data that address the continuum of oncology care.

\section{References}

1. A special report on managing information: data, data everywhere. The Economist 2010;394:3-5. 